\title{
Spectral Neugebauer-based color halftone prediction model accounting for paper fluorescence
}

\author{
Roger David Hersch \\ School of Computer and Communication Sciences, Ecole Polytechnique Fédérale de Lausanne (EPFL), \\ Switzerland 1015 Lausanne, Switzerland (RD.Hersch@epfl.ch) \\ Received 12 May 2014; accepted 22 June 2014; \\ posted 10 July 2014 (Doc. ID 208848); published 14 August 2014
}

\begin{abstract}
We present a spectral model for predicting the fluorescent emission and the total reflectance of color halftones printed on optically brightened paper. By relying on extended Neugebauer models, the proposed model accounts for the attenuation by the ink halftones of both the incident exciting light in the UV wavelength range and the emerging fluorescent emission in the visible wavelength range. The total reflectance is predicted by adding the predicted fluorescent emission relative to the incident light and the pure reflectance predicted with an ink-spreading enhanced Yule-Nielsen modified Neugebauer reflectance prediction model. The predicted fluorescent emission spectrum as a function of the amounts of cyan, magenta, and yellow inks is very accurate. It can be useful to paper and ink manufacturers who would like to study in detail the contribution of the fluorescent brighteners and the attenuation of the fluorescent emission by ink halftones. (C) 2014 Optical Society of America

OCIS codes: (100.2810) Halftone image reproduction; (260.2510) Fluorescence; (330.1710) Color, measurement.

http://dx.doi.org/10.1364/AO.53.005380
\end{abstract}

\section{Introduction}

Most white paper types incorporate fluorescent whitening agents. These whitening agents, also called optical brighteners, are needed to compensate for the slightly yellowish hue of the bleached pulp with which paper is produced. These fluorescent whitening agents absorb high-energy radiation in the ultraviolet to blue region (330-420 nm) and emit lower energy radiation mainly in the blue region of the visible spectrum $(420-530 \mathrm{~nm})$. The additional energy in the blue wavelength band counteracts the yellowish hue of the nonbrightened paper.

In traditional color management systems, no component specially accounts for the fluorescence of the paper. Fluorescence is mentioned as a reason for the lack of reproducibility when applying color management principles [1]. Therefore a color reproduction workflow is proposed where the illuminant

$1559-128 \mathrm{X} / 14 / 245380-11 \$ 15.00 / 0$

(C) 2014 Optical Society of America incorporates only very little or no UV radiation, despite the fact that removing UV light components may cause a difference in the colors perceived in a typical viewing environment $[\underline{2}, \underline{3}]$.

In this paper we present a simple and accurate spectral prediction model for color halftone prints accounting for the paper fluorescence. By measuring a limited number of patch reflectances to calibrate the model, we can predict the fluorescent emission relative to the incident light and the total reflectance of polychromatic halftones for the considered illuminant, optically brightened paper, inks, halftone method, and printing devices. Such a model may help developers in assessing the influence of paper fluorescence and decide whether it should be explicitly integrated into future color management systems. It may also help in evaluating the color gamut achievable with a given combination of illuminant, optically brightened paper, inks, and print technology. Finally, it may be helpful in determining optimal combinations of optical brighteners and inks according to given color reproduction criteria. 
We assume that the considered print samples are perfectly diffusing. We carry out reflectance factor measurements with an integrating sphere spectrophotometer, i.e., according to the $\left(\mathrm{d}: 8^{\circ}\right)$ geometry. By relying on Nicodemus et al. [4], one can show that for a Lambertian reflector, the bi-hemispherical reflectance is identical to the hemispherical directional reflectance factor (see Appendix A). Therefore, reflectances of printed samples can be obtained by simply measuring their reflectance factors. Since we consider the reflectances on small, uniformly printed areas of the samples, we express the light incident on the sample and the light reflected by the sample in terms of irradiance, i.e., radiant flux element per area element. These considerations enable us to derive the proposed prediction model by reasoning in terms of reflectances and irradiances.

\section{Related Work}

Basic work performed in the fifties and sixties of the twentieth century, related to the fluorescent brighteners and to the fluorescence of papers and inks, is summarized by Grum [5]. The Donaldson fluorescence matrix describes the emission intensities at discrete emission wavelengths as a function of the incident light intensities at discrete excitation wavelengths [6]. The deduction of the matrix coefficients requires a fluorescence spectrophotometer performing the spectral decomposition both of the illuminant and of the light reflected by the fluorescent sample.

Most fluorescent prediction models rely on the superposition paradigm; light apparently reflected by a fluorescent medium is the light reflected by that medium without fluorescence plus the light emitted by fluorescence relative to the incident light. This yields for the total reflectance $R_{\text {total }}(\lambda)$,

$$
\begin{aligned}
R_{\text {total }}(\lambda) & =\frac{I_{\mathrm{em}}(\lambda)+I_{0}(\lambda) \cdot R_{\text {pure }}(\lambda)}{I_{0}(\lambda)} \\
& =\frac{I_{\mathrm{em}}(\lambda)}{I_{0}(\lambda)}+R_{\text {pure }}(\lambda),
\end{aligned}
$$

where $I_{\mathrm{em}}(\lambda)$ is the irradiance exiting the medium that is emitted by fluorescence under incident irradiance $I_{0}(\lambda) . R_{\text {pure }}(\lambda)$ is the pure reflectance of the medium, for example obtained by successively measuring at each wavelength the reflectance using one monochromator to filter the incident light and a second monochromator to filter the reflected light (double monochromator method). It is also possible to make reflectance measurements with a set of filters that allow deducing the pure reflectance by calculation [7].

Yang [8] created a spectral prediction model accounting for the paper fluorescence in single inkhalftone prints. The model relies on Eq. (1), but assumes that the emitted fluorescent irradiance is reflected once by the paper bulk. Lateral light scattering within the paper is modeled by a probabilistic model relying on dot surface coverages and on a point-spread function. Yang [9] further extended his approach to multi-color halftone prints and to account for reflections at the print-air interface. The author does not provides any information about the prediction accuracy of these models.

Calabria and Rich [10] investigated the differences in reflectances and colors between halftones illuminated with and without UV wavelength components. They also investigated how far a classical spectral prediction model such as the Yule-Nielsen modified spectral Neugebauer model is able to model halftone prints on optically brightened paper.

A Clapper-Yule-based spectral prediction model accounting for the paper fluorescence has been previously developed by the author of the present contribution [11]. That model requires reflectance measurements of calibration patches with and without UV illumination components. In that model both the incoming UV light components and the exiting fluorescent-light components undergo multiple reflections between the paper bulk and the print-air interface.

In the present contribution we propose a simpler model relying on the combination of a simple Neugebauer model to predict the attenuation of the incident UV illumination by the ink halftones and of an ink-spreading enhanced Yule-Nielsen modified Neugebauer model to predict the spectrum emitted by fluorescence. This simpler model also provides slightly more accurate fluorescence emission prediction accuracies compared with the previous Clapper-Yule-based model.

\section{Ink-spreading Enhanced Yule-Nielsen Modified Spectral Neugebauer Model}

The most popular model for predicting the reflectances of halftone prints without specifically accounting for the fluorescence of paper is the Yule-Nielsen modified spectral Neugebauer spectral model [12-14]. It relies on the reflectances of the colorants, also called Neugebauer primaries, formed by the unprinted paper, the full-tone inks, and the superpositions of full-tone inks. The spectral reflectance $R(\lambda)$ of a color halftone is expressed as a weighted sum of colorant reflectances $R_{j}(\lambda)$, where $a_{j}$ is the area coverage of the $j$ th colorant, $R_{j}(\lambda)$ its reflection spectrum, and $n$ the Yule-Nielsen value accounting for the lateral propagation of light (in general, $1<n<100$ ), i.e., accounting for the optical dot gain:

$$
R(\lambda)=\left(\sum_{j} a_{j} R_{j}(\lambda)^{1 / n}\right)^{n} .
$$

The scalar value $n$ accounts for the lateral propagation of light in halftones and is a function of the halftone dot frequency and of the halftone dot profile. Its value is chosen so as to minimize the difference between measured and predicted reflection spectra on a limited set of halftone-model calibration samples. Note that when the $n$-value is 1 , the model becomes a pure spectral Neugebauer model, where the overall 
reflectance is the sum of the reflectances of the fulltone colorants weighted by their relative area coverages.

Hereafter, we will focus on the spectral reflectance prediction models for three inks. However, the models can be extended to four inks [15]. With three inks we have $2^{3}=8$ full-tone colorants (also called Neugebauer primaries) corresponding to all superposition combinations of $0 \%$ and $100 \%$ ink surface coverages. We assume that cyan, magenta, and yellow ink halftones are printed independently, e.g., by halftone screens mutually rotated by $30^{\circ}$. Then the area surface coverages $a_{i}$ of the colorants white $\left(a_{w}\right)$, cyan $\left(a_{c}\right)$, magenta $\left(a_{m}\right)$, yellow $\left(a_{y}\right)$, red $\left(a_{r}\right)$ (superposition of magenta and yellow), green $\left(a_{g}\right)$ (superposition of cyan and yellow), blue $\left(a_{b}\right)$ (superposition of cyan and magenta), and black $\left(a_{k}\right)$ (superposition of cyan, magenta, and yellow) are calculated according to the Demichel equations [16] expressed by Eqs. (3):

$$
\begin{aligned}
a_{w} & =(1-c)(1-m)(1-y) \\
a_{c} & =c(1-m)(1-y) \\
a_{m} & =(1-c) m(1-y) \\
a_{y} & =(1-c)(1-m) y \\
a_{r} & =(1-c) m y \\
a_{g} & =c(1-m) y \\
a_{b} & =c m(1-y) \\
a_{k} & =c m y,
\end{aligned}
$$

where $c, m$, and $y$ represent, respectively, the cyan, magenta, and yellow ink surface coverages.

This model is further enhanced by accounting for the ink-spreading phenomenon [17]. Ink-spreading accounts for the respective physical dot gain of each ink halftone dot printed in a specific superposition condition, i.e., alone on paper, in superposition with one full-tone ink, or in superposition with two fulltone inks. We therefore need to establish in each superposition condition an ink-spreading curve (or ink-spreading function) mapping nominal to effective ink surface coverages.

Effective dot surface coverages characterizing their respective ink-spreading curves are fitted separately for each superposition condition by minimizing the sum of square differences between the measured spectral reflectance components and the spectral reflectance components predicted according to the model of Eq. (2). In the case of a halftone formed by ink $i$ of effective surface coverage $u_{i / j}^{\prime}$ superposed with full-tone ink $j$, we are in the presence of a colorant dot formed by full-tone ink $j$ and of a colorant dot formed by the superpositions of full-tone inks $i$ and $j$. The effective surface coverage $u_{i / j}^{\prime}$ of this halftone dot is fitted according to the following equation:

$$
\begin{aligned}
u_{i / j}^{\prime}= & \underset{0<u^{\prime}<1}{\arg \min } \sum_{\lambda}\left(R_{m}(\lambda, u)\right. \\
& \left.-\left(\left(1-u^{\prime}\right) R_{j}^{1 / n}(\lambda)+u^{\prime} R_{i / j}^{1 / n}(\lambda)\right)^{n}\right)^{2},
\end{aligned}
$$

where $R_{m}(\lambda, u)$ is the measured reflectance of this halftone at nominal surface coverage $u, R_{j}(\lambda)$ is the measured reflectance of full-tone ink $j$, and $R_{i / j}(\lambda)$ is the measured reflectance of full-tone ink $i$ superposed with full-tone ink $j$.

By linear interpolation between fitted, effective surface coverages, one obtains for each ink halftone, and in each superposition condition, an ink-spreading curve mapping nominal to effective surface coverages (Fig. 1).

For cyan, magenta, and yellow inks with nominal surface coverages $c, m$, and $y$, the ink-spreading curves mapping nominal surface coverages to effective surface coverages for single ink halftones printed on the substrate (paper) are $f_{c}, f_{m}$, and $f_{y}$. The ink-spreading curves mapping nominal surface coverages of an ink to effective surface coverages of that ink, for single ink halftones superposed with a second full-tone ink, and for single ink halftones superposed with two full-tone inks are $f_{c / m}, f_{c / y}$, $f_{m / c}, f_{m / y}, f_{y / c}, f_{y / m}$, and $f_{c / m y}, f_{m / c y}, f_{y / c m}$, respectively, where $f_{i / j}$ indicates an ink halftone $i$ superposed with full-tone ink $j$, and where $f_{i / j k}$ indicates an ink halftone $i$ superposed with full-tone inks $j$ and $k$. In the case of three inks, these 12 ink-spreading curves may, for example, be obtained by measuring 36 uniform halftone samples, i.e., three samples (25\%, 50\%, and $75 \%$ nominal surface coverages) per curve.

For halftone reflectance prediction, one obtains the effective surface coverages $c^{\prime}, m^{\prime}$, and $y^{\prime}$ of a color halftone patch by weighting the contributions of the inkspreading curves corresponding to the occurring superposition conditions. For example, to deduce the effective cyan ink halftone surface coverage, we weight the contributions of the ink-spreading curves $f_{c}, f_{c / m}, f_{c / y}$, and $f_{c / m y}$. The weighting functions depend on the effective surface coverages of the colorants on which the considered ink halftone is superposed. Let us assume that inks are printed independently of each other. For the considered

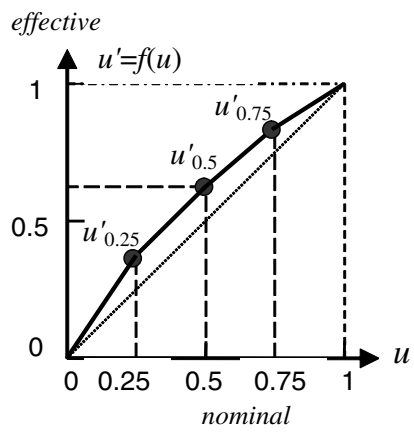

Fig. 1. Ink-spreading curve mapping nominal ink surface coverages $u$ to effective surface coverages $u^{\prime}$, where $u$ stands for $c, m$, or $y$. 
system of three cyan, magenta, and yellow inks with nominal coverages $c, m$, and $y$, and effective coverages $c^{\prime}, m^{\prime}$, and $y^{\prime}$, Eq. (5) weights each ink-spreading curve with the corresponding relative area of its underlying colorants.

In analogy with the Demichel equations [Eq. (3)], the relative weight of the cyan halftone superposed with the underlying white colorant is $\left(1-m^{\prime}\right)\left(1-y^{\prime}\right)$, the relative weight of the cyan halftone superposed with the underlying magenta colorant is $m^{\prime}\left(1-y^{\prime}\right)$, the relative weight of the cyan halftone superposed with the underlying yellow colorant is $\left(1-m^{\prime}\right) y^{\prime}$, and the relative weight of the cyan halftone superposed with the underlying red colorant is $m^{\prime} y^{\prime}$. The same line of reasoning applies to the magenta and yellow halftones. The resulting system of equations is

$$
\begin{aligned}
c^{\prime}= & f_{c}(c)\left(1-m^{\prime}\right)\left(1-y^{\prime}\right)+f_{c / m}(c) m^{\prime}\left(1-y^{\prime}\right) \\
& +f_{c / y}(c)\left(1-m^{\prime}\right) y^{\prime}+f_{c / m y}(c) m^{\prime} y^{\prime}, \\
m^{\prime}= & f_{m}(m)\left(1-c^{\prime}\right)\left(1-y^{\prime}\right)+f_{m / c}(m) c^{\prime}\left(1-y^{\prime}\right) \\
& +f_{m / y}(m)\left(1-c^{\prime}\right) y^{\prime}+f_{m / c y}(m) c^{\prime} y^{\prime}, \\
y^{\prime}= & f_{y}(y)\left(1-c^{\prime}\right)\left(1-m^{\prime}\right)+f_{y / c}(y) c^{\prime}\left(1-m^{\prime}\right) \\
& +f_{y / m}(y)\left(1-c^{\prime}\right) m^{\prime}+f_{y / c m}(y) c^{\prime} m^{\prime} .
\end{aligned}
$$

This system of equations can be solved iteratively. One starts by setting initial values of $c^{\prime}, m^{\prime}$, and $y^{\prime}$ equal to the respective nominal ink surface coverages $c, m$, and $y$. After one iteration, one obtains new values for $c^{\prime}, m^{\prime}$, and $y^{\prime}$. These new values are used for the next iteration. After a few iterations, typically four to five, the system stabilizes and the obtained coverages $c^{\prime}, m^{\prime}$, and $y^{\prime}$ are the effective coverages. Equation (드) yields the effective surface coverages of cyan, magenta, and yellow inks for the corresponding nominal surface coverages.

\section{Overview of the Proposed Print Fluorescence Model}

As in previous research approaches $[8, \underline{9}, \underline{11}, 18]$, the proposed paper fluorescence model incorporates both the attenuation of the incoming excitation light components in the UV wavelength range and the

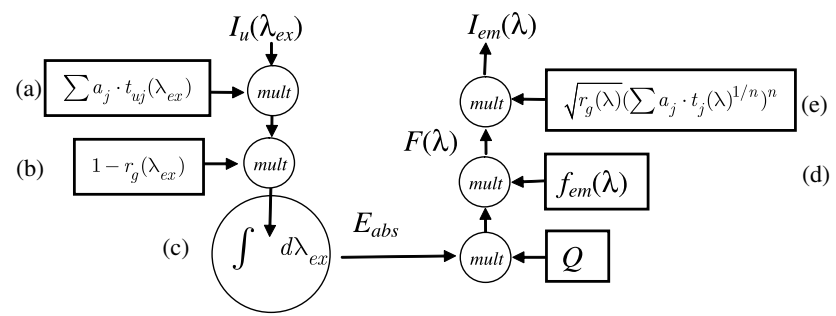

Fig. 2. Print fluorescence model. (a) Attenuation of incident light $I_{u}\left(\lambda_{\text {ex }}\right)$ in the UV excitation wavelength range by the ink halftone, (b) absorption, (c) conversion into energy $E_{\text {abs, }}$, (d) energy release by fluorescent emission $F(\lambda)=E_{\text {abs }} Q \cdot f_{\text {em }}(\lambda)$, and (e) attenuation by the paper and the ink halftone and resulting emerging emission $I_{\mathrm{em}}(\lambda)$ in the visible wavelength range. attenuation of the induced fluorescent emission by the printed ink halftones. Figure 2 provides an overview of the scheme on which the presently proposed paper fluorescence model relies. For the case of three inks and eight full-tone colorants (Neugebauer primaries), the component $I_{u}\left(\lambda_{\text {ex }}\right)$ of the incident light within the excitation wavelength range (UV wavelength range) is attenuated by the multi-ink halftone expressed as Neugebauer halftone transmittance $\sum_{j=1}^{8} a_{j} \cdot t_{u j}\left(\lambda_{\mathrm{ex}}\right)$, where $t_{u j}\left(\lambda_{\mathrm{ex}}\right)$ expresses the transmittance of colorant $j$ in the excitation wavelength range (UV). Within the paper bulk, a part $r_{g}(\lambda)$ of the exciting irradiance transmitted by the halftone is reflected toward the surface and a part $\left(1-r_{g}(\lambda)\right)$ is absorbed by the optically brightened paper whose fluorescent molecules reach a higher energy state. This energy is partly released by fluorescent emission $F(\lambda)$ in the visible wavelength range. The emission intensity depends on the absorbed irradiance and on the efficiency $Q$ of releasing the absorbed energy $E_{\text {abs }}$ as fluorescent emission.

The fluorescent emission $F(\lambda)$ in the visible wavelength range is then partially attenuated by the paper and by one traversal across the ink halftone before exiting from the paper. In the classical case of light reflection on a halftone print, light incident on the print traverses the ink halftone, is reflected by the paper bulk, and traverses again the ink halftone [attenuation $r_{g}(\lambda) \sum_{j=1}^{8} a_{j} \cdot t_{j}(\lambda)^{2}$ ]. The subsequent multiple reflections of part of the light between the print-air interface and the paper bulk as well as the lateral propagation of light are accounted for by the Yule-Nielsen $n$-value [see Eq. (2)]. The resulting halftone reflectance is

$$
R=r_{g}(\lambda)\left(\sum_{j=1}^{8} a_{j} \cdot t_{j}(\lambda)^{2 / n}\right)^{n} .
$$

In the case of a fluorescent emission, the emitted light emerges from the paper bulk and traverses the ink halftone only once. Therefore, its attenuation is modeled by $\sqrt{r_{g}(\lambda)} \cdot \sum_{j=1}^{8} a_{j} \cdot t_{j}(\lambda)$, where $r_{g}(\lambda)$ is the reflectance of paper and $t_{j}(\lambda)$ the transmittance of full-tone colorant $j$ in the visible wavelength range. The subsequent multiple reflections between the print-air interface and the paper bulk and the lateral propagation of part of the light are accounted for by performing the ink surface coverage dependent weighting of the colorant transmittances in the spectral attenuation space $T(\lambda)^{1 / n}$ [19]. The resulting attenuation of the fluorescent emission emerging from the print is

$$
\sqrt{r_{g}(\lambda)}\left(\sum_{j=1}^{8} a_{j} \cdot t_{j}(\lambda)^{1 / n}\right)^{n} .
$$

The emitted irradiance $I_{\text {em }}(\lambda)$ (see Fig. 2) is added to the irradiance $I_{r}(\lambda)$ reflected by the print by pure reflectance, forming the emerging irradiance $I_{\text {out }}(\lambda)$. This emerging irradiance divided by the incident irradiance $I_{0}(\lambda)$ yields the total reflectance $R_{\text {total }}(\lambda)$. 


\section{Fluorescent Emission of Optically Brightened Paper Across Printed Halftones}

The fluorescence model is an extension of the classical Yule-Nielsen modified spectral Neugebauer model [12,13] enhanced with superpositiondependent ink spreading [17]. We consider separately the attenuation of the incoming light in the excitation wavelength range. We assume that the incident light in the excitation wavelength range (UV) passes once through the attenuating ink halftone layer and is partially absorbed by the optically brightened paper. Light is attenuated differently by each colorant (Neugebauer primary) forming the halftone. The resulting attenuation of the incident light in the excitation wavelength range of the fluorescent brighteners is the attenuation $t_{u j}\left(\lambda_{\text {ex }}\right)$ of each colorant $j$, weighted by its relative surface coverage $a_{j}$. With $I_{u}\left(\lambda_{\text {ex }}\right)$ expressing the incident irradiance in the excitation wavelength range $\lambda_{\text {exInf }}<\lambda_{\text {ex }}<\lambda_{\text {exSup }}$ we obtain for the absorbed part $I_{\text {abs }}\left(\lambda_{\text {ex }}\right)$ of the incident light in the excitation wavelength range

$$
I_{\mathrm{abs}}\left(\lambda_{\mathrm{ex}}\right)=I_{u}\left(\lambda_{\mathrm{ex}}\right) \cdot\left(\sum_{j} a_{j} \cdot t_{u j}\left(\lambda_{\mathrm{ex}}\right) \cdot\left(1-r_{g}\left(\lambda_{\mathrm{ex}}\right)\right),\right.
$$

where $r_{g}(\lambda)$ is the paper reflectance. By expressing the paper absorption as $\alpha\left(\lambda_{\text {ex }}\right)=1-r_{g}\left(\lambda_{\text {ex }}\right)$, and by integrating the absorbed light over the excitation wavelength range $\left[\lambda_{\text {exInf }}, \lambda_{\text {exSup }}\right]$, we obtain a measure of absorbed power per surface element:

$$
E_{\mathrm{abs}}=\int_{\lambda_{\mathrm{exInf}}}^{\lambda_{\mathrm{exSup}}} I_{u}(\lambda) \cdot\left(\sum_{j} a_{j} \cdot t_{u j}(\lambda) \cdot \alpha(\lambda) \cdot \mathrm{d} \lambda .\right.
$$

We can perform the weighting by the wavelength independent ink halftone surface coverages $a_{j}$ outside the integral

$$
E_{\mathrm{abs}}=\sum_{j} a_{j} \int_{\lambda_{\mathrm{exInf}}}^{\lambda_{\mathrm{exSup}}} I_{u}(\lambda) \cdot t_{u j}(\lambda) \cdot \alpha(\lambda) \cdot \mathrm{d} \lambda .
$$

The integral within Eq. (8) expresses the power per surface element $E_{\text {abs } J}(\lambda)$ absorbed after attenuation $t_{u j}(\lambda)$ of the excitation light by full-tone colorant $j$. This attenuation can be approximated using an equivalent scalar colorant attenuation $t_{u j}^{\prime}$ :

$$
\begin{aligned}
E_{\text {abs } J} & =\int_{\lambda_{\text {exInf }}}^{\lambda_{\text {exSup }}} I_{u}(\lambda) \cdot t_{u j}(\lambda) \cdot \alpha(\lambda) \cdot \mathrm{d} \lambda \\
& \cong t_{u j}^{\prime} \int_{\lambda_{\text {exInf }}}^{\lambda_{\text {exSup }}} I_{u}(\lambda) \cdot \alpha(\lambda) \cdot \mathrm{d} \lambda .
\end{aligned}
$$

For the unprinted paper, there is no colorant attenuation. Therefore the absorbed power per surface element of the unprinted paper is

$$
E_{\mathrm{abs} P}=\int_{\lambda_{\mathrm{exInf}}}^{\lambda_{\mathrm{exSup}}} I_{u}(\lambda) \cdot \alpha(\lambda) \cdot \mathrm{d} \lambda .
$$

The fluorescent emission spectrum $F(\lambda)$ of light travelling upward from the fluorescent paper bulk is located within the visible wavelength range and is proportional to the absorbed power $E_{\text {abs, }}$, with the proportionality constant $Q$ indicating the fraction of absorbed power re-emitted by fluorescence. The shape of the normalized fluorescent emission spectrum $f_{\mathrm{em}}(\lambda)$, nonzero in the visible wavelength range, depends on the fluorescent brighteners added to the paper. Within the visible wavelength range $\left[\lambda_{v \operatorname{Inf}}\right.$, $\left.\lambda_{v \text { Sup }}\right]$, the normalized emission spectrum $f_{\text {em }}(\lambda)$ is defined as

$$
\int_{\lambda_{v} \mathrm{Inf}}^{\lambda_{v \mathrm{Sup}}} f_{\mathrm{em}}(\lambda) \cdot \mathrm{d} \lambda \equiv 1
$$

The fluorescent emission spectrum $F(\lambda)$ emitted by the optically brightened paper bulk is proportional to $f_{\text {em }}(\lambda)$ and to the absorbed power $E_{\text {abs. }}$. According to Eqs. ( 1$)$ and (9), it can be expressed as the absorption power per surface element $E_{\text {abs } J}$ of the individual print colorants weighted by their surface coverages:

$$
F(\lambda)=E_{\mathrm{abs}} \cdot Q \cdot f_{\mathrm{em}}(\lambda)=Q \cdot f_{\mathrm{em}}(\lambda) \cdot\left(\sum a_{j} \cdot E_{\mathrm{abs} J}\right) .
$$

The emitted light ready to emerge from the print is the fluorescent emission spectrum $F(\lambda)$ attenuated by a one-way travel through the paper bulk (attenuation $\left.\sqrt{r_{g}(\lambda)}\right)$ and through the colorants $t_{j}(\lambda)$ forming the ink halftone. Fresnel reflections at the print-air interface and lateral propagation of light within the paper bulk are accounted for by the Yule-Nielsen $n$-value [13].

For a halftone composed of colorants having transmittances $t_{j}(\lambda)$ and surface coverages $a_{j}$, we obtain for the emitted irradiance

$$
I_{\mathrm{em}}(\lambda)=F(\lambda) \cdot \sqrt{r_{g}(\lambda)}\left(\sum_{j}\left(a_{j} \cdot t_{j}(\lambda)^{1 / n}\right)\right)^{n} .
$$

Thanks to Eqs. (12), (9)), and (10) the emerging emitted irradiance can be further expressed as

$$
\begin{aligned}
I_{\mathrm{em}}(\lambda)= & E_{\mathrm{abs} P} \cdot Q \cdot f_{\mathrm{em}}(\lambda) \\
& \cdot\left(\sum_{j}\left(a_{j} \cdot t_{u j}^{\prime}\right)\right) \sqrt{r_{g}(\lambda)}\left(\sum_{j}\left(a_{j} t_{j}(\lambda)^{1 / n}\right)\right)^{n} .
\end{aligned}
$$

\section{Prediction of Relative Emission Irradiance and Total Reflectance}

The total reflectance $R_{\text {total }}(\lambda)=R_{\mathrm{UV}+V}(\lambda)$ given by Eq. (1) can be measured with an illuminant also incorporating components within the excitation 
wavelength range (UV), for example, the standard D65 illuminant or its approximations provided in high-quality commercial spectrophotometers. The pure reflectance $R_{\text {pure }}(\lambda)=R_{V}(\lambda)$ present in Eq. ( $\left.\underline{1}\right)$ can also be measured in the visible wavelength range only by inserting a filter eliminating the excitation components from the illuminant. For example, the Gretag-Macbeth i7 desktop spectrophotometer offers such a capability called "UV excluded" measurement mode.

From Eq. (1) we deduce a relationship between measured total reflectance, measured pure reflectance, and the emitted irradiance $I_{\mathrm{em}}$ normalized by the incident irradiance $I_{0}$ :

$$
R_{\mathrm{UV}+V}(\lambda)-R_{V}(\lambda)=\frac{I_{\mathrm{em}}(\lambda)}{I_{0}(\lambda)} .
$$

From Eq. (14) we deduce

$$
\begin{aligned}
\frac{I_{\mathrm{em}}(\lambda)}{I_{0}(\lambda)}= & E_{\mathrm{abs} P} Q \frac{f_{\mathrm{em}}(\lambda)}{I_{0}(\lambda)} \\
& \cdot\left(\sum_{j}\left(a_{j} t_{u j}^{\prime}\right)\right) \sqrt{r_{g}(\lambda)}\left(\sum_{j}\left(a_{j} t_{j}(\lambda)^{1 / n}\right)\right)^{n} .
\end{aligned}
$$

Within Eq. (16) the pure paper reflectance $r_{g}(\lambda)=$ $R_{p V}(\lambda)$ is obtained by a spectral reflectance measurement of the unprinted paper with a light source excluding UV components.

In order to obtain the transmittances $t_{j}(\lambda)$ of each colorant $j$, we make the following assumptions: in a full-tone colorant print of reflectance $R_{j V}(\lambda)$, the incident light is attenuated by the paper reflectance $r_{g}(\lambda)$ and by twice the transmittance $t_{j}(\lambda)$ of its colorant. Therefore, the colorant transmittance $t_{j}(\lambda)$ in the visible wavelength range is deduced from the full-tone colorant print reflectances $R_{j V}(\lambda)$ measured in a UV-excluding mode according to the equation

$$
t_{j}(\lambda)=\sqrt{\frac{R_{j V}(\lambda)}{r_{g}(\lambda)}} .
$$

Note that $t_{j}(\lambda)$ represents the attenuation of diffuse light traversing the ink layer. It is not identical to the regular transmittance of the ink, measured perpendicularly to the print surface.

The effective surface coverages $a_{j}$ of the colorants are obtained by first establishing at calibration time the nominal to effective ink-surface coverage curves in the different superposition conditions in a UVexcluding mode (see Section 3). At prediction time for a given color halftone of nominal surface coverages, the effective surface coverage of each ink is obtained by weighting the contributing nominal to effective surface coverage curves according to the surface coverages of the other superposed ink dots [see Eq. (5)]. Then, by applying the Demichel equations [Eq. (3)], one obtains the effective surface coverages $a_{j}$ of the colorants forming that color halftone.

Let us now show how to obtain the unknown elements of Eq. (16), namely the equivalent scalar colorant attenuations $t_{u j}^{\prime}$ in the excitation wavelength range (UV) as well as the relative fluorescent emission spectrum $I_{\mathrm{em} P}(\lambda) / I_{0}(\lambda)$ emerging from the unprinted paper. According to Eq. (16), by setting all surface coverages of the ink colorants to zero and the unprinted paper colorant surface coverage to one, i.e., $a_{j \neq p}=0$ and $a_{j=p}=1$, and since the unprinted paper does not attenuate the incident or emerging irradiance [for unprinted paper, $t_{u j}^{\prime}=1$ and $t_{j}(\lambda)$ is 1 at every wavelength], we obtain

$$
\frac{I_{\mathrm{em} P}(\lambda)}{I_{0}(\lambda)}=E_{\mathrm{abs} P} \cdot Q \cdot \frac{f_{\mathrm{em}}(\lambda)}{I_{0}(\lambda)} \sqrt{r_{g}(\lambda)} .
$$

The relative emission spectrum $I_{\mathrm{em} P}(\lambda) / I_{0}(\lambda)$ emerging from the unprinted paper is obtained by measuring, according to Eq. (15), the reflectance of the unprinted paper with and without UV components. We insert the corresponding measurements as $R_{p \mathrm{UV}+V}(\lambda)$ and $R_{p V}(\lambda)$ in Eq. (15), where subscript $p$ stands for paper. We obtain from Eqs. (15) and (18)

$$
\begin{aligned}
R_{p \mathrm{UV}+V}(\lambda)-R_{p V}(\lambda) & =\frac{I_{\mathrm{em} P}(\lambda)}{I_{0}(\lambda)} \\
& =\frac{E_{\mathrm{abs} P} \cdot Q \cdot f_{\mathrm{em}}(\lambda)}{I_{0}(\lambda)} \cdot \sqrt{r_{g}(\lambda)} .
\end{aligned}
$$

In order to obtain the equivalent scalar colorant attenuations $t_{u j}^{\prime}$ of the colorants in the excitation wavelength range (UV), we consider Eq. (16) for each full-tone colorant $k$. For a single colorant $k, a_{k}$ is 1 , and all other $a_{j}$ are zero. We obtain

$$
\begin{aligned}
R_{k \mathrm{UV}+V}(\lambda)-R_{k V}(\lambda) & =\frac{I_{\mathrm{em} K}(\lambda)}{I_{0}(\lambda)} \\
& =E_{\mathrm{abs} P} Q \frac{f_{\mathrm{em}}(\lambda)}{I_{0}(\lambda)} \sqrt{r_{g}(\lambda)} \cdot t_{u k}^{\prime} \cdot t_{k}(\lambda) .
\end{aligned}
$$

By inserting the right part of Eq. (19) into (20) we obtain

$$
R_{k \mathrm{UV}+V}(\lambda)-R_{k V}(\lambda)=\left(R_{p \mathrm{UV}+V}(\lambda)-R_{p V}(\lambda)\right) \cdot t_{k}(\lambda) \cdot t_{u k}^{\prime} .
$$

Since $t_{k}(\lambda)$ is known from Eq. (17), by minimizing the left part minus the right part of Eq. (21), we can fit the optimal value of the scalar colorant attenuation $t_{u k}^{\prime}$

$$
\begin{aligned}
t_{u k}^{\prime}= & \underset{0 \leq t_{u k}^{\prime} \leq 1}{\arg \min } \sum_{\lambda=380 \mathrm{~nm}}^{730 \mathrm{~nm}}\left[\left(R_{k \mathrm{UV}+V}(\lambda)-R_{k V}(\lambda)\right)\right. \\
& \left.-t_{u k}^{\prime} \cdot\left(R_{p \mathrm{UV}+V}(\lambda)-R_{p V}(\lambda)\right) \cdot t_{k}(\lambda)\right]^{2} .
\end{aligned}
$$


Now that all unknowns of Eq. (16) have been obtained by Eqs. (19) and (22), we can reformulate Eq. (16) for the prediction of the emerging fluorescent irradiance $I_{\mathrm{em}}(\lambda)$ relative to the incident irradiance $I_{0}(\lambda)$ :

$$
\begin{aligned}
\frac{I_{\mathrm{em}}(\lambda)}{I_{0}(\lambda)}= & \left(R_{p \mathrm{UV}+V}(\lambda)-R_{p V}(\lambda)\right) \\
& \cdot\left(\sum_{j}\left(a_{j} t_{u j}^{\prime}\right)\right)\left(\sum_{j}\left(a_{j} t_{j}(\lambda)^{1 / n}\right)\right)^{n} .
\end{aligned}
$$

According to Eq. (1), the total reflectance of color halftones can be predicted by adding the relative emerging fluorescent emission irradiance given by Eq. $(23)$ to the predicted pure reflectance $R_{V}(\lambda)$, see Eq. (24). The predicted pure reflectance $R_{V}(\lambda)$ relies on the ink-spreading enhanced Yule-Nielsen modified spectral Neugebauer model [17]. That model is calibrated by reflectance measurements with an illuminant excluding UV components, see Section $\underline{3}$ :

$$
\begin{aligned}
R_{\mathrm{UV}+V}(\lambda)= & \left(R_{p \mathrm{UV}+V}(\lambda)-R_{p V}(\lambda)\right) \\
& \cdot\left(\sum_{j}\left(a_{j} t_{u j}^{\prime}\right)\right)\left(\sum_{j}\left(a_{j} t_{j}(\lambda)^{1 / n}\right)\right)^{n}+R_{V}(\lambda) .
\end{aligned}
$$

\section{Calibration of the Model and Experimental Verification}

All measured spectral reflectances are obtained with a Gretag-Macbeth i7 integrating sphere spectrophotometer, where diffuse incident light illuminates the print and where the reflected light is captured at $8^{\circ}$ (geometry: di: $8^{\circ}$ ) with the specular component being included. Two illuminants are available: one that includes and one that excludes most of the UV light components. The corresponding illuminant spectra are shown in Fig. 3.

Note that for eliminating the fluorescence effect induced by fluorescent whitening agents, ISO

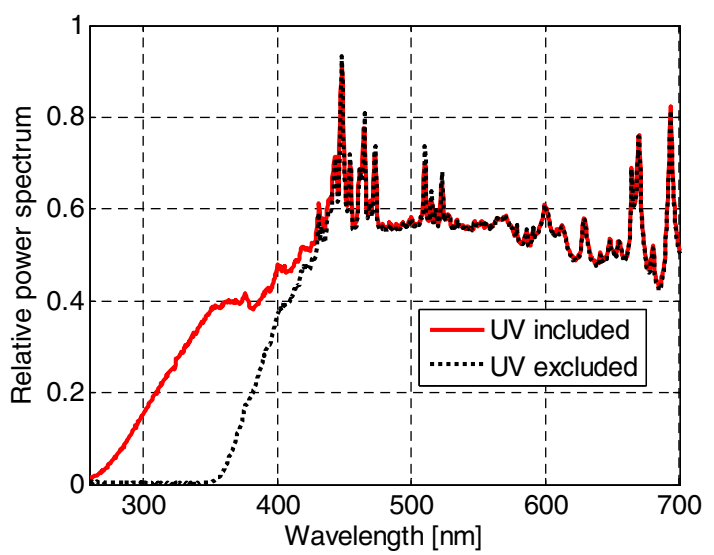

Fig. 3. Power spectra of the two illuminants, UV included and UV excluded, present in the Gretag-Macbeth i7 spectrophotometer, captured by a MayaPro Ocean Optics spectrophotometer.
Standard 2469 recommends a sharp cut-off filter at $420 \mathrm{~nm}$ (halfpeak cut-off wavelength).

In order to compute colors or color differences, reflection spectra are converted to CIE-XYZ tristimulus values with a D65 standard illuminant and according to the CIE $19312^{\circ}$ standard observer. CIE-XYZ values are then converted to the CIELAB color space with the paper white as reference white. The color patches are printed with a Canon Pro 9500 inkjet printer on a Canon MP-101 matte optically brightened paper, at a screen frequency of $120 \mathrm{lpi}$ and a resolution of $600 \mathrm{dpi}$, with classical mutually rotated clustered dot screens.

\section{A. Prediction Model Calibration}

The model is calibrated by measuring the spectral reflectances of all full-tone colorants with UV illumination included and excluded. For the present experiment with cyan, magenta, and yellow inks printed on optically brightened paper, we have eight colorants that need to be measured with and without UV components. This yields 16 spectral measurements. For the calibration of the 12 ink-spreading curves, with measurements at surface coverages of $0.25,0.5$, and 0.75 we need 36 additional spectral measurements with UV illumination excluded (see Section 3). With a total of $16+36=52$ spectral measurements, the three ink spectral-prediction model accounting for paper fluorescence can be completely calibrated. An $n$-value of 38 was found to be optimal for predicting the reflectances of the color halftones with the IS-YNSN model, both for calibrations in UV-excluding and UV-including modes.

In order to obtain the seven ink colorant attenuation factors $t_{u j}^{\prime}$, we first compute the colorant transmittances $t_{j}(\lambda)$ with UV excluded according to Eq. (17), by making use of the unprinted paper reflectance $r_{g}(\lambda)=R_{p V}(\lambda)$ measured also in a UVexcluding mode. Then, for the nonwhite colorants $j$, we fit the colorant attenuation factor $t_{u j}^{\prime}$ according to Eq. (22), for every nonwhite colorant $k=j$.

\section{B. Impact of the Optical Brighteners on the Reflectances} of Paper and Full-Tone Inks

Let us first qualitatively examine the impact of the fluorescent brighteners on the paper white and on the full-tone colorant prints. Figure 4 clearly shows that for the unprinted optically brightened paper white, there is a very strong difference in reflectance if the brightened paper is illuminated with light comprising components in the excitation wavelength range (UV) or not (CIELAB difference $\Delta E_{94}=10.4$ ). The fluorescent emission has a smaller impact for the full-tone cyan and magenta prints (cyan, $\Delta E_{94}=3.7$; magenta, $\Delta E_{94}=3.6$ ). For the yellow print, there is nearly no fluorescent emission $\left(\Delta E_{94}=0.67\right)$. We presume that the strong decrease in fluorescent emission of the full-tone prints is mainly due to the absorption of the excitation light by the inks and to some extent due to the attenuation of the light emitted by the fluorescent emission of 


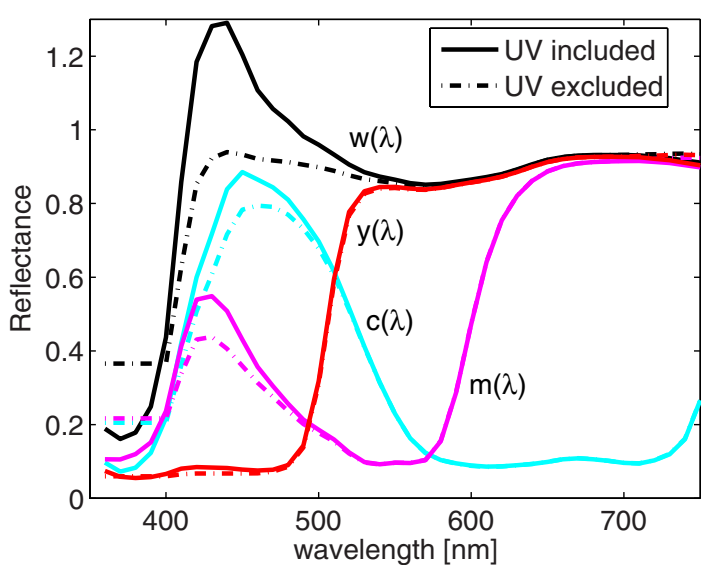

Fig. 4. Measured reflectances of paper white (w) and of full-tone cyan $(\mathrm{c})$, magenta $(\mathrm{m})$, and yellow (y) patches under illuminants with and without UV component, as a function of wavelength. Reflectances with UV included and excluded represent the total and pure reflectances, respectively.

paper. The strong reduction in fluorescent emission in the case of the full-tone colorants suggests that fluorescent brighteners incorporated into the paper have the greatest impact on white and on highlight colors created by halftone dots of small and mid-size ink surface coverages.

\section{Experimental Setup for the Accuracy Tests}

The present section aims at verifying the accuracy of the spectral prediction model accounting for paper fluorescence and at analyzing to what extent the ink-spreading enhanced Yule-Nielsen modified spectral Neugebauer model [17], which does not specifically account for paper fluorescence, might be sufficient to predict the color of halftones printed on optically brightened paper.

Tests are carried out on a first set of 125 colored test samples measured both with UV included and UV excluded. This set (full set, FS,) comprises all possible combinations of surface coverages of the cyan, magenta, and yellow inks at surface coverages 0 , $0.25,0.5,0.75$, and 1 . Tests are also carried out on a second set of 81 test samples (TS, test set), both with UV included and excluded, formed by the samples of the first set minus the $8+36$ samples used for the calibration of the model. Further tests are carried out on a third set (LS, low-surface coverage test set) which comprises the 20 samples having combinations of low ink-dot surface coverages of $0,0.25$, and 0.5 without including any calibration sample. The color halftones of this LS test set have a surface coverage of unprinted paper white of at least 0.125 . Therefore, they exhibit a significant fluorescent behavior.

The tests comprise (a) the accuracy of the emission spectra predictions under UV illumination, (b) the accuracy of the total reflectance prediction under UV illumination, and (c) for comparison purposes, the accuracy that is achieved by using the inkspreading enhanced Yule-Nielsen modified spectral Neugebauer model ink-spreading enhanced
Table 1. Colorant Attenuation Factors $t_{u j}^{\prime}$ in the Excitation Wavelength Range Fitted According to Eq. (22)

Colorant Cyan Magenta Yellow Red Green Blue Black

\begin{tabular}{llllllllll}
\hline$t_{u j}^{\prime}$ & & 0.3853 & 0.4394 & 0.1798 & 0.1387 & 0.1351 & 0.2439 & 0.1401 \\
\hline
\end{tabular}

Yule-Nielsen modified spectral Neugebauer model (IS-YNSN) directly on the spectral measurements under UV illumination.

The colorant attenuation factors $t_{u j}^{\prime}$ in the UV wavelength range are given in Table 1 . The stronger the attenuation, the lower the resulting fluorescent emission, expressed by the total reflectance minus the pure reflectance, see Fig. $\underline{4}$.

\section{Accuracy of the Spectral Emission Prediction}

The relative fluorescent emission $I_{c m y \mathrm{Em}}(\lambda) / I_{0}(\lambda)$ of a given sample of ink surface coverages $c, m$, and $y$ can be obtained by the measurements of $R_{c m y \mathrm{UV}}(\lambda)$ (UV included) and $R_{c m y V}(\lambda)$ (UV excluded) according to Eq. (15). It can also be predicted according to Eq. (23), with colorant surface coverages calculated by first obtaining the effective ink surface coverages $c^{\prime}, m^{\prime}, y^{\prime}$ according to Eq. (5), and then the effective colorant surface coverages $a_{j}$ according to Eq. (3).

Table 2 (first row) gives the average, $95 \%$ quantile, and maximal CIELAB $\Delta E_{94}$ error as well as the average rms difference characterizing the spectral emission prediction for the first test set (FS) incorporating all 125 samples, the second test set (TS) of 81 samples excluding the calibration samples, and for the third test set comprising 20 halftones with individual ink surface coverages $u_{j} \leq 0.5$, also excluding the calibration samples. The $\Delta E_{94}$ error has been calculated between the measured total reflectance and the predicted total reflectance obtained by adding the predicted relative fluorescent emission and the measured pure reflectance. All CIELAB values are calculated with the pure reflectance $r_{g}(\lambda)$ of paper as adapting white reference.

E. Accuracy of the Total Reflectance Prediction Obtained by Adding the Predicted Fluorescent Emission and the Pure Reflectance Predicted with the IS-YNSN Model

The total reflectance prediction is obtained according to Eq. (24) by separately (a) predicting the pure reflectance $R_{V}(\lambda)$ with the IS-YNSN model under UV excluded light and (b) predicting the relative fluorescent emission. Table $\underline{2}$ (second row) gives the average, $95 \%$ quantile, and maximal CIELAB $\Delta E_{94}$ error for the total reflectance prediction for the three test sets.

F. Accuracy of the Total Reflectance Prediction Obtained with the IS-YNSN Model Only, Calibrated on Samples Measured Under an Illumination with UV Components

As an alternative to the presented fluorescent emission prediction model, one may simply calibrate the IS-YNSN model with measurements carried out on the samples measured with UV included, and then predict all other samples. Table 2 (third row) gives 
Table 2. Prediction Accuracy Given by Average, $95 \%$ Quantile, and Maximal CIELAB $\Delta E_{94}$ Prediction Error and by Average RMS Difference for Three Data Sets Under the Illuminant Including UV Components (Fig. 3) ${ }^{a}$

\begin{tabular}{|c|c|c|c|c|c|c|}
\hline & & & \multicolumn{4}{|c|}{$\Delta E_{94}$ Prediction Error } \\
\hline & & & Average & $95 \%$ Quantile & Max & Average rms Difference \\
\hline \multirow[t]{3}{*}{ Spectral Emission Prediction (New Model) } & FS & 125 samples & 0.310 & 0.532 & 0.661 & 0.0026 \\
\hline & $\mathrm{TS}$ & 81 samples & 0.325 & 0.574 & 0.661 & 0.0026 \\
\hline & LS & 20 samples & 0.335 & 0.532 & 0.573 & 0.0036 \\
\hline \multirow[t]{3}{*}{ Total Reflectance Prediction (New Model) } & FS & 125 samples & 0.764 & 1.727 & 2.032 & 0.0054 \\
\hline & $\mathrm{TS}$ & 81 samples & 0.934 & 1.758 & 2.032 & 0.0064 \\
\hline & LS & 20 samples & 1.208 & 1.898 & 2.032 & 0.0090 \\
\hline Total Reflectance Prediction with & $\mathrm{FS}$ & 125 samples & 0.724 & 1.734 & 2.126 & 0.0060 \\
\hline \multirow[t]{2}{*}{ IS-YNSN Model Only } & $\mathrm{TS}$ & 81 samples & 0.957 & 1.819 & 2.126 & 0.0080 \\
\hline & $\mathrm{LS}$ & 20 samples & 1.157 & 1.941 & 2.126 & 0.0129 \\
\hline
\end{tabular}

${ }^{a}$ The n-value is 38 .

Table 3. For Reference, Pure Reflectance Prediction Accuracy by the IS-YNSN Model Given by Average, $95 \%$ Quantile, and Maximal CIELAB $\Delta E_{94}$ Prediction Error and by Average RMS Difference for the 125 Sample, the 81 Sample and the 20 Sample Sets Under the Illuminant Excluding UV Components (Fig. 3)

\begin{tabular}{|c|c|c|c|c|c|c|}
\hline & & & \multicolumn{4}{|c|}{$\Delta E_{94}$ Prediction Error } \\
\hline & & & Average & 95\% Quantile & $\operatorname{Max}$ & Average rms Difference \\
\hline \multirow[t]{3}{*}{ Pure Reflectance Prediction (IS-YNSN) } & FS & 125 samples & 0.735 & 1.857 & 2.072 & 0.0055 \\
\hline & $\mathrm{TS}$ & 81 samples & 0.968 & 1.893 & 2.072 & 0.0073 \\
\hline & LS & 20 samples & 1.236 & 2.012 & 2.072 & 0.0109 \\
\hline
\end{tabular}

the average, 95\% quantile, and maximal CIELAB $\Delta E_{94}$ error for the total IS-YNSN-based reflectance prediction for the three test sets.

As a reference we also give in Table 3 the prediction accuracies of the IS-YNSN model for the different sample sets illuminated by the light source excluding the UV components. This reference gives an idea of the prediction accuracies that may be attained in cases where there is no fluorescent emission from the optical brighteners.

\section{G. Discussion of the Prediction Results}

The prediction of the fluorescent spectral emission of the color halftones is extremely accurate with a predicted average error $\Delta E_{94}=0.34$ for fluorescent samples having a minimal surface coverage of paper of 1/8 (Table 2, first row, LS sample set). The prediction relies on the relative emission intensity of the unprinted paper obtained from measurements according to Eq. (15), on the attenuation of the incident excitation light by the ink halftones in the excitation wavelength range, and on the attenuation of the emerging fluorescent emission by the same halftones in the visible wavelength range, see Eq. (23).

The prediction of the total reflection by the model accounting for paper fluorescence and by the prediction of the pure reflection is also accurate, but less than the prediction of the spectral emission only (average error on the 81 samples of the TS sample set, $\left.\Delta E_{94}=0.93\right)$. This decrease in prediction accuracy is due to the fact that the total reflectance prediction requires two separate models whose predicted spectra are added, namely, the emission prediction and the IS-YNSN model reflectance prediction with the UV excitation components excluded. In terms of rms difference, the total reflectance prediction accuracy of the new model accounting for the optically brightened paper is similar or slightly better than the accuracy of the state of the art IS-YNSN model serving as reference (compare Table 2 , second row, last column, and Table 3 last column).

Finally, we observe that carrying out total reflectance predictions with the prior art IS-YNSN model [17] calibrated according to Section 3 on printed color samples illuminated by a light source including UV components yields reflectance spectra predictions, which are slightly less accurate in terms of the rms difference than the ones predicted with the paper fluorescence model (compare Table 2, last column, third row and second row). However, from a colorimetric point of view, the predictions are equivalent, sometimes slightly worse and sometimes slightly better, than the predictions with the paper fluorescence accounting model.

\section{Conclusions}

We propose a new model for predicting the emission of halftone patches printed on paper with fluorescent brighteners. The calibration of the model is performed by measuring the reflectances of the unprinted optically brightened paper and of the printed full-tone colorants under an illuminant with excitation components (UV) included and under the same illuminant with excitation components (UV) excluded. With excitation components included, the total reflectance is measured and with excitation 
components excluded, the pure reflectance is measured. The difference between the measured total reflectance and the measured pure reflectance of the unprinted optically brightened paper expresses the relative fluorescent emission under the considered illuminant incorporating excitation components.

On a full-tone colorant print, this fluorescent emission is reduced due to the specific attenuation of the incident light within the excitation wavelength range by that colorant. We therefore introduce for each colorant a scalar colorant attenuation factor that is fitted by minimizing a difference metric between its predicted and its measured relative fluorescent emission. This colorant attenuation factor characterizes how much a full-tone ink or a superposition of full-tone inks attenuates the incident excitation light components.

Within the visible wavelength range, the fluorescent emission emerging from the optically brightened paper is attenuated by the printed colorant. This attenuation is modeled by the square root of the paper reflectance multiplied by the transmittance of the colorant.

In the case of halftone prints, the partial attenuation of the incident light in the excitation wavelengths is the weighted average of the attenuations of the individual colorants, the weights being formed by the colorant halftone surface coverages. In the visible wavelength range, the attenuation of the fluorescent emission is modeled by weighting the colorant attenuations in transmission mode according to the ink-spreading enhanced Yule-Nielsen modified spectral Neugebauer model [19].

The fluorescent emission prediction model provides accurate predictions of the fluorescent emission with an average prediction error of $\Delta E_{94}=0.32$. Total reflectance predictions are obtained by adding the predicted relative fluorescent emission spectra and the predicted pure reflectances obtained according to the ink-spreading enhanced Yule-Nielsen modified spectral Neugebauer model. Average total reflection prediction accuracy is $\Delta E_{94}=0.93$.

As an alternative to a framework capable of predicting separately the fluorescent emission of printed optically brightened paper, one may also use the well-known ink-spreading enhanced Yule-Nielsen modified spectral Neugebauer model [17] to predict the total reflectance by calibrating it on samples printed on optically brightened paper, measured with an illuminant incorporating UV components. In this case, average total reflection prediction accuracy is $\Delta E_{94}=0.96$.

Therefore, for simple total reflection predictions, the ink-spreading enhanced Yule-Nielsen modified spectral Neugebauer model is sufficient. However, for manufacturers of inks and of optically brightened paper, the presented fluorescent emission prediction model may be of significant value. It enables separating the different factors contributing to the total reflectance of halftone print samples, such as the incident light attenuation in the UV wavelength range and the attenuation of the fluorescent emission by the halftone within the visible wavelength range.

In the future we may extend this model so as to predict the fluorescent emission under a variety of light sources without needing to perform the full calibration separately for each light source.

\section{Appendix A}

Let us establish the equivalence between reflectance and reflectance factors for a reflector (e.g., paper) assuming to be perfectly diffusing. The following derivations rely on the definitions and formula proposed by Nicodemus et al. [4]. The hemisphericaldirectional reflectance factor $\bar{R}$ obtained with a $\left(d: 0^{\circ}\right)$ measurement geometry is given by

$$
\begin{aligned}
\bar{R}\left(2 \pi, \theta_{r}, \phi_{r}\right)= & \int_{0}^{2 \pi} \int_{0}^{\pi / 2} f_{r}\left(\theta_{i}, \phi_{i}, \theta_{r}, \phi_{r}\right) \\
& \times \cos \theta_{i} \sin \theta_{i} \mathrm{~d} \theta_{i} \mathrm{~d} \phi_{i},
\end{aligned}
$$

where $\left(\theta_{i}, \phi_{i}\right)$ express the direction of the incident light and $\left(\theta_{r}, \phi_{r}\right)$ the direction of the captured light, and where $f_{r}$ expresses the bi-directional reflectance distribution function (BRDF).

A perfectly diffusing reflector (Lambertian reflector) has a constant BRDF. The corresponding reflectance factor becomes

$$
\bar{R}\left(2 \pi, \theta_{r}, \phi_{r}\right)=f_{r} \int_{0}^{2 \pi} \int_{0}^{\pi / 2} \cos \theta_{i} \sin \theta_{i} \mathrm{~d} \theta_{i} \mathrm{~d} \phi_{i}=f_{r} \cdot \pi .
$$

The bi-hemispherical reflectance is expressed by

$$
\begin{aligned}
R\left(2 \pi, \theta_{r}, \phi_{r}\right)= & (1 / \pi) \cdot \int_{0}^{2 \pi} \int_{0}^{\pi / 2}\left(\int_{0}^{2 \pi} \int_{0}^{\pi / 2} f_{r}\left(\theta_{i}, \phi_{i}, \theta_{r}, \phi_{r}\right)\right. \\
& \left.\times \cos \theta_{i} \sin \theta_{i} \mathrm{~d} \theta_{i} \mathrm{~d} \phi_{i}\right) \cos \theta_{r} \sin \theta_{r} \mathrm{~d} \theta_{r} \mathrm{~d} \phi_{r} .
\end{aligned}
$$

For the Lambertian reflector, the bi-hemispherical reflectance becomes

$$
\begin{aligned}
R\left(2 \pi, \theta_{r}, \phi_{r}\right)= & (1 / \pi) \cdot f_{r}\left(\theta_{i}, \phi_{i}, \theta_{r}, \phi_{r}\right) \int_{0}^{2 \pi} \int_{0}^{\pi / 2} \int_{0}^{2 \pi} \int_{0}^{\pi / 2} \\
& \times \cos \theta_{i} \sin \theta_{i} \mathrm{~d} \theta_{i} \mathrm{~d} \phi_{i} \cos \theta_{r} \sin \theta_{r} \mathrm{~d} \theta_{r} \mathrm{~d} \phi_{r} \\
= & (1 / \pi) \cdot f_{r} \cdot \pi \cdot \pi=f_{r} \cdot \pi .
\end{aligned}
$$

This shows that for a Lambertian reflector, measuring a reflectance factor with a hemisphericaldirectional device (e.g., Gretag-Macbeth i7 spectrophotometer) is equivalent to measuring its bi-hemispherical reflectance.

In addition, since the bi-directional reflectance factor is 


$$
\bar{R}\left(\theta_{i}, \phi_{i}, \theta_{r}, \phi_{r}\right)=\pi \cdot f_{r}\left(\theta_{i}, \phi_{i}, \theta_{r}, \phi_{r}\right)
$$

a reflectance factor measurement on a Lambertian reflector with a $\left(45^{\circ}: 0^{\circ}\right)$ geometry is also equivalent to the measurement of a bi-hemispherical reflectance.

Many thanks to Romain Rossier for having measured the print samples and to Thomas Walger for proofreading the paper. The project has been partly funded by the Swiss National Science Foundation, grants 200020-105119 and 200021-143501.

\section{References}

1. CIE Publication 163 (ICC Summary), "The effects of fluorescence in the characterization of imaging media," ICC White paper \#14, 2005, http://www.color.org/.

2. ISO 13655:2009, "Graphic technology-Spectral measurement and colorimetric computation for graphic arts images," http://www.iso.org/iso/catalogue_detail?csnumber=39877.

3. ICC Recommendations for Color Measurement, White Paper \#3, 2004, http://www.color.org/.

4. F. E. Nicodemus, J. C. Richmond, J. J. Hsia, I. W. Ginsberg, and T. Limperis, "Geometrical considerations and nomenclature for reflectance," National Bureau of Standards, US Dept. of Commerce (1977), http://graphics.stanford.edu/courses/cs44805-winter/papers/nicodemus-brdf-nist.pdf.

5. F. Grum, "Colorimetry of fluorescent materials," in Optical Radiation Measurements, F. Grum and C. J. Bartelson, eds., Vol. 2 of Color Measurements (Academic, 1980), pp. 235-288.

6. R. Donaldson, "Spectrophotometry of fluorescent pigments," Br. J. Appl. Phys. 5, 210-214 (1954).

7. E. Allen, "Separation of the spectral radiance factor curve of fluorescent substrates into reflected and fluoresced components," Appl. Opt. 12, 289-293 (1973).

8. L. Yang, "Spectral model of halftone on a fluorescent substrate,” J. Imaging Sci. Techn. 49, pp. 179-184 (2005).
9. L. Yang, "Probabilistic spectral model of color halftone incorporating substrate fluorescence and interface reflections," J. Opt. Soc. Am. A 27, 2115-2122 (2010).

10. A. J. Calabria and D. C. Rich, "Brighter is better? Investigating spectral color prediction of ink on optically brightened substrate," in Proceedings of IS\&T/SID 11th Color Imaging Conference (IS\&T, 2003), 288-293.

11. R. D. Hersch, "Spectral prediction model for color prints on paper with fluorescent additives," Appl. Opt. 47, 6710-6722 (2008).

12. H. E. J. Neugebauer, "Die theoretischen Grundlagen des Mehrfarbendrucks," Zeitschrift fuer wissenschaftliche Photographie 36, 36-73 (1937) [translated by D. Wyble and A. Kraushaar, "The theoretical basis of multicolor letterpress printing," Color Res. Appl. 30, 323-331 (2005)].

13. J. A. S. Viggiano, "Modeling the Color of Multi-Colored Halftones," in Technical Association of the Graphic Arts Proceedings (1990) pp. 44-62.

14. R. Balasubramanian, "Optimization of the spectral Neugebauer model for printer characterization," J. Electron. Imaging 8, 156-166 (1999).

15. T. Bugnon, M. Brichon, and R. D. Hersch, "Simplified inkspreading equations for CMYK halftone prints," Proc. SPIE 6807, 680717 (2008).

16. D. R. Wyble and R. S. Berns, "A critical review of spectral models applied to binary color printing," Color Res. Appl. 25, 4-19 (2000).

17. R. D. Hersch and F. Crété, "Improving the Yule-Nielsen modified spectral Neugebauer model by dot surface coverages depending on the ink superposition conditions," Proc. SPIE 5667, 434-445 (2005).

18. G. W. Gill, "A practical approach to measuring and modelling paper fluorescence for improved colorimetric characterization of printing processes," in Proceedings of Imaging Science \& Technology / Society for Information Display 11th Color Imaging Conference (IS\&T, 2003) pp. 248-254.

19. M. Hébert and R. D. Hersch, "Yule-Nielsen based recto-verso color halftone transmittance prediction model," Appl. Opt. 50, 519-525 (2011). 